\title{
CCD Photometry of the Weakly Barred Triple-Ringed Galaxy IC 4214
}

\author{
M. F. Saraiva \\ Department of Astronomy and McDonald Observatory, University of \\ Texas, Austin, TX 78712; and Departamento de Astronomia, Instituto \\ de Física, Universidade Federal do Rio Grande do Sul, Brazil
}

\begin{abstract}
We present a brief report on the $B, V, R, I$, and $\mathrm{H} \alpha \mathrm{CCD}$ surface photometry of the southern SABab galaxy IC 4214. We obtained isophote maps, luminosity profiles, and basic geometric and photometric parameters in $B V R I$, and mapped the distribution of the gas through continuum subtracted $\mathrm{H} \alpha$ emission line images.
\end{abstract}

\section{Introduction}

The southern galaxy IC 4214, classified as $\left(\mathrm{R}^{\prime}\right) \mathrm{SB}(\mathrm{r}) \mathrm{ab}$ in the RC3 (de Vaucouleurs et al. 1991), is an example of a weakly barred galaxy with the three main ring types possible in a galaxy: a nuclear ring $(9.5 \times 8.5$ arcsec $)$, an inner ring ( $52 \times 27$ arcsec), and an outer ring ( $118 \times 73$ arcsec). The three rings were previously shown in the $B-I$ map by Buta \& Crocker (1991).

The inner and outer ring are connected by two enhanced regions located in both extremities of the inner ring. The weak bar is not clearly distinct from the inner ring, appearing as a mixture of the types "ring/lens" barlike distortion and "spiral" distortion (Buta 1986). The morphology of the galaxy is illustrated in Figure 1.

\section{Observations}

The observations were made in June 1992 using the TEK 1024 CCD at the $91 \mathrm{~cm}$ telescope of the Cerro Tololo Inter-American Observatory (CTIO). The filters used were $B, V, R, I$, and two narrow filters of $75 \AA$ wide centered at $6649 \AA$ (to isolate the $\mathrm{H} \alpha$ emission line at the redshift of the galaxy) and at $6477 \AA$ (to isolate a nearby region of stellar continuum).

\section{Results}

The total apparent magnitude and color of IC 4214, obtained from photoelectrically calibrated synthesized elliptical apertures, are $\mathrm{m}(B)=12.25$ and $B-V$ $=0.88$. After correcting by Galactic and internal absorption the corresponding absolute magnitude is $M_{T}^{o}(B)=-20.44$ (based on the radial velocity corrected 


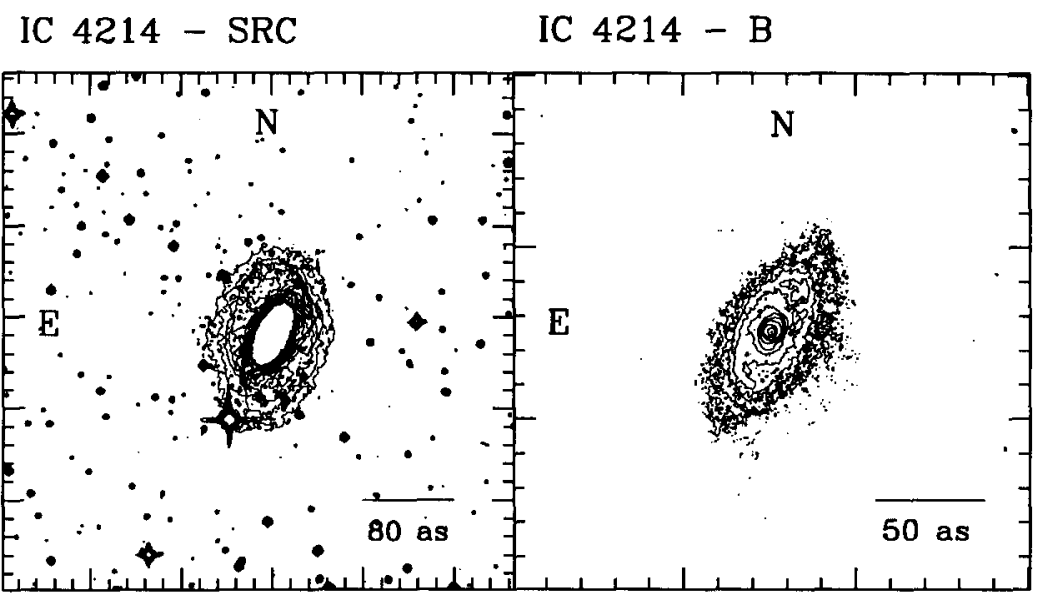

Figure 1. Isophote maps of IC 4214. Left: based on the digitized SRC plate; shows the isophotes external to the inner ring. Right: based on our CCD $B$ image; shows the isophotes internal to the outer ring.

to the Galactic Standard of Rest in RC3, V $=2135 \mathrm{~km} \mathrm{~s}^{-1}$, and assuming $H=$ $85 \mathrm{~km} \mathrm{~s}^{-1} \mathrm{Mpc}^{-1}$ ). The effective equivalent radius is $r_{e}^{*}=21 \operatorname{arcsec}=2.6 \mathrm{kpc}$.

Ellipse fits to the isophotes show that the inner ring is the most elongated feature in the galaxy; the nuclear ring is almost round, and nearly parallel to the inner ring; the outer ring has the same orientation and ellipticity of the bulge. The bulge is not spherical, showing distorted oval-rectangular isophotes and asymmetric luminosity profiles in the direction of the major axis of the inner ring. The color profiles are very noisy, indicating the presence of dust in the bulge region. The existence of the dust appears more clearly in the mean $B-I$ profile, where it makes a strong bump centered at $r=12^{\prime \prime}$. In spite of the dust, the mean profile obtained from the ellipse fits follows an $r^{1 / 4}$ law (de Vaucouleurs 1948) in the bulge region ( $\mathrm{r}<16$ arcsec).

A Fourier analysis with a sinusoidal basis shows that the $m=2$ and $m=4$ components have maxima in the surroundings of the inner ring, reinforcing the existence of a bar or oval in this region. These two components contribute $21 \%$ of the $B$ and $I$ luminosity within $r=80^{\prime \prime}$.

Continuum-subtracted $H \alpha$ emission line images (Figure 2) show that the emission is concentrated at the nucleus, the nuclear ring and the two spiral arms that form the inner ring. The nuclear ring is complete in $\mathrm{H} \alpha$, but in the $B-I$ map it appears as an arc-shaped feature located in the Northeast side of the galaxy center. The colors of the nuclear ring $\left[(B-V)^{\circ}=0.72,(V-R)^{\circ}=\right.$ $\left.0.53,(V-I)^{\circ}=1.1\right]$ indicate it contains star formation regions. The connection between nuclear rings of star formation and bars (Arsenault 1989; Pogge 1989) is another indication of the existence of a barred structure in IC 4214 . 


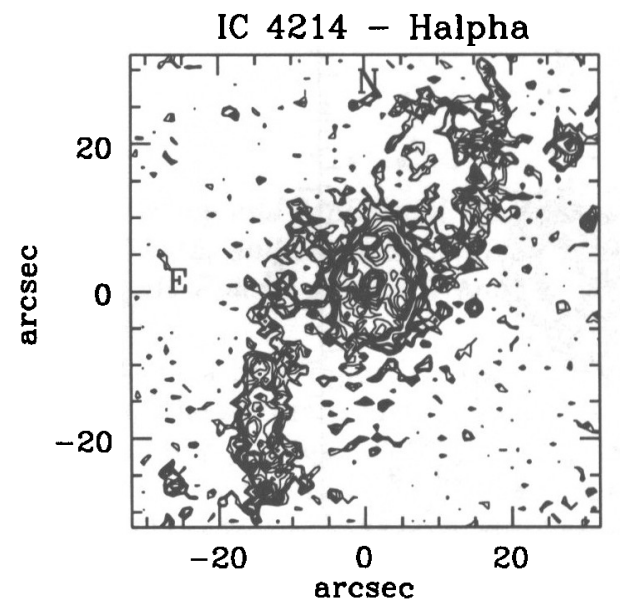

Figure 2. $\mathrm{H} \alpha$ map of the inner region of IC 4214, based on the continuum-subtracted $\mathrm{H} \alpha$ emission line images.

Acknowledgments. I would like to thank Gerard de Vaucouleurs for his wise suggestions in the beginning of this work, Steve Odewahn for kindly providing me with his Numerical Mapping Technique code and helping me with its use, Miriani Pastoriza for suggesting this work to me, and Ron Buta for a helpful discussion. I acknowledge the Brazilian agency $\mathrm{CNPq}$ for a fellowship.

\section{References}

Arsenault, R. 1989, A\&A, 217, 66

Buta, R. 1986, ApJS, 61, 609

Buta, R. \& Crocker, D. 1991, AJ, 102, 1715

de Vaucouleurs, G. 1948, Ann.d' Ap., 11, 247

de Vaucouleurs, G., de Vaucouleurs, A., Corwin, H., Buta, R., Paturel, G., \& Fouqué, P. 1991, Third Reference Catalogue of Bright Galaxies, New York: Springer-Verlag (RC3)

Pogge, R. 1989, ApJS, 71, 433 